\title{
Craniodigital-intellectual disability syndrome
}

INSERM

\section{Source}

INSERM. (1999). Orphanet: an online rare disease and orphan drug data base.

Craniodigital-intellectual disability syndrome. ORPHA:1514

Craniodigital syndrome - intellectual deficit is characterised by syndactyly of the fingers and toes, characteristic facies ('startled' facial expression with a small pointed nose, micrognathia, long dark eyelashes and prominent eyebrows) and intellectual deficit. 